EDUCATION

Research, Innovation and Solutions on-line
PSYCHOLOGY I+D+i
Electronic Journal of Research

in Educational Psychology

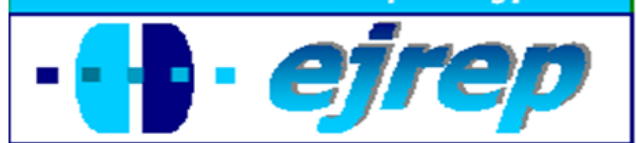

Editorial EOS

\title{
El desempeño en habilidades sociales en niños, de dos y tres años de edad, y su relación con los estilos de interacción parental
}

Laura Isaza Valencia y Gloria Cecilia Henao López

Facultad de Psicologìa, Universidad San Buenaventura, Medellín

\section{Colombia}

Correspondencia: Laura Isaza Valencia. Calle 40 N. 81 A 118 apto 102. Edificio Galeón. Barrio Simón Bolivar. Medellín, Antioquia. Colombia. E-mail: isaza8888@hotmail.com

(C) Education \& Psychology I+D+i and Editorial EOS (Spain) 


\section{Resumen}

Introducción: Esta investigación tuvo como objetivo estudiar los estilos de interacción familiar y el desarrollo de habilidades sociales en niños y niñas de dos y tres años de edad. Se consideró el desarrollo social como un proceso educativo gradual, en donde las acciones que ejercen los padres son la base para potenciar el desarrollo social de sus hijos e hijas.

Método: Se evaluaron las acciones de los padres desde los estilos de interacción autoritario, equilibrado y permisivo, y el desempeño social mediante los seis repertorios conductuales. La investigación fue de tipo descriptivo correlacional. Se trabajó con una muestra de 108 niños y niñas y sus respectivas familias.

Resultados: Se encontró que los estilos autoritario y permisivo presentes en los padres se asocian con bajo nivel de habilidades sociales, mientras que el estilo de interacción equilibrado propicia la presencia de repertorios conversacionales, de interacción, solución de problemas y asertividad.

Discusión y Conclusiones: Los resultados de este estudio aportan información relevante y pertinente sobre los factores asociados al comportamiento de niños y niñas desde la dimensión social, lo cual es punto de partida para la creación y aplicación de programas de formación para los padres. El constructo estilo de interacción parental conlleva un amplio e importante análisis por la variedad de elementos que involucra y su relación en el desarrollo de los niños y las niñas a nivel del desempeño social.

Palabras Clave: Contexto familiar, prácticas educativas familiares, desarrollo social, conducta sociales, repertorios básicos sociales.

Recibido: 16/06/10 Aceptación Inicial: 29/06/10 Aceptación Definitiva: 18/10/10 


\title{
Social skills performance in two- and three-year-olds and its relationship with parental interaction styles
}

\begin{abstract}
Introduction: This investigation aimed to study the styles of familiar interaction and the development of social skills in children of two and three years of age. Consider a contribution for social development is a gradual learning process, where parents exert actions that are the basis for enhancing the social development of their sons and daughters.
\end{abstract}

Method: The actions of the parents were evaluated from the styles of authoritarian interaction, permissive balance and, and it carried out social by means of the six behavioural repertoires. The investigation was of correlational descriptive type. One worked with a sample of 108 children and children and their respective families.

Results: One was that the styles authoritarian and permissive presents in the parents are associated with low level of social abilities, whereas the sensible style of interaction causes the presence of conversational repertoires, of interaction, solution of problems and assertiveness.

Discussion and Conclusions: The results of this study contribute to relevant and pertinent information on the factors associated to the behavior of children and children from the social dimension, which is departure point for the creation and application of programs of formation for the parents. Constructo style of parental interaction entails an ample and important analysis by the variety of elements that it involves and its relation in the development of the children and the children concerning the social performance

Keywords: Familiar context, family educational practices, social development, social behavior, basic social codes.

Received: 06/16/10 Initial Acceptance: 06/29/10 Definitive Acceptance: 10/18/10 


\section{Introducción}

El concepto educación se amplia a diferentes aspectos de la vida, considerándose un proceso permanente, que abarca tanto la educación familiar como la educación escolar. Pero, la relación escuela-familia para potenciar las influencias educativas es sólo reconocida en el plano teórico, existiendo dificultades que estriban en la materialización de la colaboración entre estos contextos y del protagonismo de la familia. Los padres por diversas razones desplazan la responsabilidad educativa a la escuela; ya que, con la obligatoriedad de la escolarización y el carácter instructivo adjudicado a la escuela se privilegió la misión educativa de esta. El papel y el protagonismo de la familia ha quedado disminuido en lo que se refiere a las prácticas de enseñanza-aprendizaje, cuando en realidad la familia y las relaciones que existen en ellas están intervenidas por una misión pedagógica, en la que los padres educan a sus hijos e hijas en estrategias fundamentales para que logren aprendizajes significativos.

El estudio de la familia como contexto educativo parte de los presupuestos contextualista y social-culturalista; estos permiten analizar el papel que desempeña el sistema familiar como contexto de socialización de los niños y las niñas. La propuesta contextualista (Eisenberg, Valiente, Morris, Fabes, Cumberland y Reiser, 2003; Forgas, 2000; Sroufe, 2000) valora la experiencia social del sujeto para acceder a los aprendizajes, resalta a los padres como fuente socializadora y agentes modeladores para sus hijos e hijas. Sus acciones y verbalizaciones posibilitan un aprendizaje y adquisición de repertorios de interacción social y lenguaje, y de habilidades emocionales y cognitivas. Según el modelo social-culturalista; la familia, más específicamente los padres y adultos que rodean al niño y a la niña, llevan a estos y a estas a progresar en el aprendizaje (Rogoff, 1993; Vigotsky, 1979). Desde sus acciones (verbales y no verbales) y sus relaciones, les posibilitan aprendizajes de repertorios sociales al brindarles conocimientos y estrategias acerca del mundo circundante. Estas interacciones con sus padres y adultos cercanos permiten el alcance de competencias en las diversas dimensiones (cognitiva, comunicativa, socio-afectiva, corporal, estética, espiritual y ética). Este modelo reconoce la participación guiada de los padres en el proceso de adquisición de aprendizaje de los hijos e hijas y resalta las interacciones presentes entre los adultos y los infantes como el proceso necesario que permite pasar de una zona de desarrollo real a una zona de desarrollo proximal; el niño o la niña logra hacer una actividad en compañía del adulto, y conseguir posteriormente desplegar la capacidad de solucionar independientemente esa actividad. 
La familia como el contexto básico de desarrollo humano cumple una acción socializadora en el niño y la niña, los padres y adultos llevan a cabo esta a través del modelamiento y del tipo de comunicación que establecen con los niños y las niñas; la acción socializadora se dirige al alcance de metas y pautas comportamentales mediante el control y el afecto usado por los padres y adultos en las diversas situaciones (Henao y García, 2009). Para estas autoras, es en el entorno familiar donde se establecen "prácticas educativas, que se convierten en punto de referencia para niños y niñas, permitiendo el logro de estilos de socialización, el desarrollo de competencias emocionales, el manejo de estrategias de afrontamiento y niveles de prosocialidad, entre otros" (p. 787).

Las prácticas educativas familiares o estilos de interacción parental como fueron denominos en éste trabajo de investigación, han sido señalados por diversos auotores como elementosde importancia en la eduación de la primera infancia (Bronfenbrenner, 1987; Coll, 1988; Solé, 1997; Vila, 1998). Estos estilos son abordados como facilitadores y estimuladores del proceso de desarrollo en el niño y la niña a nivel social, en la medida que los preparan para implantarse en la sociedad. Es por medio de los estilos de interacción parental que se logra distinguir la dinámica propia de cada familia, estos se evidencian en el pensamiento y en las creencias de los padres o adultos que rodean al niño y la niña respecto a elementos como la norma, la autoridad, el afecto, el control y la comunicación. Al hablar de estos estilos, Ceballos y Rodrigo (1998) señalan las prácticas más frecuentes utilizadas por los padres o los adultos que están en contacto directo y continuo con los niños y las niñas, estas se presentan de manera flexible y son seleccionadas por los padres de acuerdo a sus particularidades, a las características del hijo o la hija, a la situación y a los factores externos. Es así, como éstos estilos se manifiestan en diversos contextos, siendo como mencionan Coll, Miras, Onrubia y Solé (1998) exclusivos y concretos de cada familia, pero a su vez, equivalentes a los que manejan otras familias que participan en el mismo grupo social.

Son muchos los autores que han abordado los estilos de interacción parental, García y Román (2003) se han destacado en sus investigaciones por el abordaje de esta dimensión familiar. Ellos reconocen éstos como las estrategias con las que los padres intentan provocar en el hijo y la hija la inhibición o inducción de la conducta. Estos investigadores trabajan estas prácticas desde tres estilos: autoritario (mantiene valores bajos en la expresión de afecto y comunicación, y alto en cuanto a exigencias y control), equilibrado (presenta valores altos en expresión de afecto y comunicación, pero altos también en exigencias y control; con una dis- 
ciplina inductiva y sensible a las necesidades de los hijos e hijas) y permisivo (crianza sobreprotectora, con alto grado de expresión explícita de afecto, pero poco consistente en sus normas de disciplina). El estilo de interacción parental es un proceso fundamental en la construcción de valores en familia, propiamente en la adquisición de valores prosociales, de autodirección y al logro de aprendizajes significativos en los niños y las niñas (Garcia, Ramirez y Lima, 1998; García y Román, 2003).

Los trabajos de García y Román $(2003,2005)$ van en la misma dirección de estudios que enfatizan en la asociación presente entre el estilo educativo familiar y la autoestima de los niños y las niñas, dependiendo del estilo de interacción de los padres, los hijos y las hijas estructuran un tipo de apego seguro, inseguro o ambivalente y la posibilidad de un bienestar psicológico (Asili y Pinzón, 2003). Por su parte, Fernández (2003) en su estudio, resalta cómo los padres desde los distintos tipos de paternidad contribuyen de manera positiva en el desarrollo cognoscitivo y socio-emocional en estos niños y niñas. Por último, Mestre, Tur, Samper, Nácher y Cortés (2007) quienes investigaron los estilos de crianza en la adolescencia y su relación con el comportamiento prosocial, concluyen la alta correlación que existe entre los estilos de crianza con la empatía, la agresividad, la inestabilidad emocional y la ira desde su doble planteamiento.

Estudiar el desarrollo social infantil se debe tener en cuenta factores de tipo contextual referidos a las circunstancias de vida que posean los niños y las niñas, y las interacciones padres-niño/a, como un componente clave del contexto, ya que logran convertirse en un modelador. Los padres y los adultos mediante los estilos de interacción parental propician el desarrollo de habilidades sociales, éstas son las capacidades o destrezas sociales específicas requeridas para ejecutar competentemente una tarea interpersonal, han sido llamadas de diversas formas: habilidades sociales, habilidades de interacción social, habilidades para la interacción, habilidades interpersonales, habilidades de relación interpersonal, habilidades para la relación interpersonal, destrezas sociales, habilidades de intercambio social, conducta interactiva, conducta interpersonal, relaciones interpersonales, conducta sociointeractiva, intercambios sociales, entre otros (Caballo, 1993; Elliot y Gresham, 1991; Hundert, 1995; Sorkhabi, 2005; Vallejo, Aguilar y Valencia, 2002). Estas habilidades hacen referencia al grado en que el niño y la niña han adquirido una serie de repertorios comportamentales que permiten su adaptación a las exigencias del medio social. Se enumeran tanto las relacionadas con auto- 
nomía e independencia personal como las que tienen que ver con la capacidad de relación interpersonal.

En la familia las acciones cotidianas se convierten en prácticas de enseñanzaaprendizaje, de acuerdo a como se estructuren permiten un avance en el desarrollo de conductas prosociales en los niños y las niñas, o por el contrario se presenta el desarrollo y el mantenimiento de conductas disfuncionales. Los factores familiares como las fallas en la disciplina temprana e inadecuada supervisión de las actividades, aumentan en los niños y las niñas los riesgos de comportamiento agresivo que en algunos casos llevan a la delincuencia en la adolescencia y la adultez, esto se hace evidente en los resultados arrojados en diversos estudios (Florsheim, Tolan y Gorman-Smith, 1996; Patterson, Reid y Dishion, 1992; Stoolmiller, 2001). Además, la calidad de la relación marital es un agente relacionado con la conducta social de los niños y niñas y el ajuste emocional infantil y adolescente (Ballesteros, 1995; Chang y Dodge, 2003; Cowan, Cohn, Cowan y Pearson, 1996; Eisenberg, Losoya, Fabes, Guthrie, Reiser, Murphy, Shepard, Poulin y Padgett, 2001; Rogers Y Holmberck, 1997; Shaw, Keenan y Vondra, 1994).

Esto permite resaltar el interés de esta investigación, la cual se propuso estudiar aquellos aspectos que interfieren o afectan de manera positiva el desempeño en habilidades sociales en niños y niñas, de manera más concreta los estilos de interacción parental que pueden propiciar el desarrollo o no de repertorios sociales. Este estudio, permite continuar reforzando las ideas existentes frente a la importancia de la familia, y la creación de espacios de interacción con ellas para su formación, en donde se exalte la familia como el principal contexto de socialización. Se refuerza en la familia su rol director y coordinador de importantes factores influyentes en el desarrollo social.

\section{Método}

\section{Participantes}

Se tomó una muestra de 108 niños y niñas, entre dos y tres años, de edad de nivel socioeconómico bajo, medio y alto de la ciudad de Medellín y sus respectivas familias (Tabla 1). Este grupo estuvo conformado por 54 niñas (50\%) y 54 niños (50\%), distribuidos en dos 
grupos de edad teniendo el $62 \%$ de los niños y las niñas 2 años de edad y el $41 \% 3$ años de edad. Los niños y las niñas pertenecían a los tres niveles socioeconómicos, divididos de la siguiente manera: 35 niños y niñas pertenecen a un nivel socioeconómico bajo $(32,4 \%), 37$ a un nivel medio $(34,3 \%)$ y 36 a un nivel alto (33,3\%). Por último, de los 108 niños y niñas, el $66,7 \%$ se encontraban escolarizados, mientras el otro $33,3 \%$ no estaba cursando ningún nivel educativo.

Tabla 1. Características sociodemográficas de la muestra

\begin{tabular}{lcc}
\hline & Frecuencia & $\%$ \\
\hline Género & & \\
$\quad$ Masculino & 54 & 50 \\
$\quad$ Femenino & 54 & 50 \\
Edad & & \\
2 años & 67 & 62 \\
3 años & 41 & 38 \\
Nivel socioeconómico & & \\
Bajo & 35 & 32,4 \\
Medio & 37 & 34,3 \\
Alto & 36 & 33,3 \\
Escolaridad & & \\
Si & 72 & 66,7 \\
No & 36 & 33,3 \\
\hline
\end{tabular}

\section{Instrumentos}

Para la evaluación de los estilos de interacción familiar se utilizó la Prueba de Prácticas Educativas Familiares (PEF) de García y Román (2003), esta contiene una escala para adultos, la cual recoge la percepción de los padres, madres, tutores o responsables encargados de la educación de los niños y las niñas pequeños en el entorno familiar. Las percepciones se recogen a través de tres situaciones: Cuando inician algo nuevo, cuando hay una ruptura de rutinas, cuando cuentan o muestran algo. Se obtiene un puntaje total para cada estilo parental: autoritario, equilibrado y permisivo. Cada situación se evalúa mediante 18 ítems para un total de 54. Esta escala ha sido construida y validada por García y Román (2003) en España, los alfas de Cronbach en cada factor se presentaron de la siguiente manera: Estilo autoritario padres 0.85 , madres 0.85 , estilo equilibrado padres 0.87 , madres 0.89 , estilo permisivo padres 0.83 y madres 0.81 . El Alfa de Cronbach de la prueba total es de 0.86 . 
Las habilidades sociales fueron evaluadas mediante el instrumento creado por Monjas (2000) en el "Programa de enseñanza de HHSS". Este instrumento fue sometido a una prueba piloto y a juicios de expertos. Este instrumento lo diligenciaron los padres y madres de los niños y niñas evaluados, a partir 53 items en los que se evaluó la ausencia y presencia de cada conducta y su frecuencia. Este instrumento evalúa seis habilidades sociales a partir de las conductas que las constituyen: Habilidades básicas de interacción social (sonreir, reir, saludar, presentaciones, favores, cortesía y amabilidad), habilidades para hacer amigos y amigas (reforzar a otros, iniciaciones sociales, unirse a juegos de otros, ayuda, cooperar y compartir), habilidades conversacionales (iniciar conversaciones, mantener conversaciones, terminar conversaciones, unirse a conversaciones de otros, conversaciones en grupo), habilidades relacionadas con los sentimientos, emociones y opiniones (auto-afirmaciones positivas, expresar emociones, recibir emociones, defender los proios derechos, defender las opiniones propias), habilidades de solución de problemas interpersonles (identificar problemas interpersonles, buscar soluciones, anticipar consecuencias, elegir una solución, probar solución) y habilidades para relacionarse con los adultos (cortesía con los adultos, refuerzo al adulto, conversar con el adulto, peticiones al adulto y solucionar problemas con adultos). El instrumento permité evaluar el desempeño social de los niños y niñas en este rango de edad, se hace desde el análisis de los datos una lectura del desarrollo y el contexto. El Alfa de Cronbach de la prueba total es de 0.82 .

\section{Procedimiento}

- Selección de la muestra: El marco muestral se estableció a través de un sorteo para alcanzar unproceso aleatorio estratificado, el cual se realizó en hogares de bienestar familiar, guardería, iglesias, juntas de acción comunal y bibliotecas lúdicas de los tres niveles socioeconómicos de la ciudad de Medellín. Para un total de 108 niños y niñas entre dos y tres años de edad y sus respectivas familias.

- Elaboración o prueba de los instrumentos: Se realizó una selección de las pruebas que permitían evaluar las variables del estudio. Se realizó pilotaje y juicio de expertos del Cuestionario de Habilidades sociales de Monjas (2000), con la finalidad de revisar que el vocabulario original de esta escala se adecue al contexto de la cultura colombiana. 
- Motivación y vinculación de las familias: Se le informó a la comunidad de padres y madres de familia seleccionados en el proceso investigativo, lo concerniente a lo metodológico, el tipo de pruebas que se utilizarían, el tiempo invertido por ellos y los resultados esperados de la investigación

- Aplicación de instrumentos: Se realizó en los hogares los cuestionarios en compañía de los auxiliares de investigación.

\section{Diseño y análisis estadístico}

Esta investigación fue de tipo no experimental, trasversal y correlacional, ya que se caracterizaron los estilos de interacción parental identificados en un grupo de 108 familias y el desempeño en habilidades sociales de 54 niños y 54 niñas entre dos y tres años de edad; además, se comparó las variables de estudios por género, edad y nivel socioeconómico; y se estudió las relaciones presentes entre el estilo de interacción parental y habilidades sociales. Las familias pertenecían a los tres niveles socio-económicos de la ciudad de Medellín.

Los análisis realizados en este estudio para describir la variable habilidades sociales fueron las medidas de tendencia central (medias, desviación estándar y percentiles) considenrando la edad y el género de los niños y niñas. Se realizó la prueba t para comparar las habilidades sociales teniendo en cuenta el género. Y se realizó un análisis de Spearman para correlacionar las habilidades sociales con los estilos de interacción parental.

\section{Resultados}

\section{Estilos de interacción familiar}

Se observa que en las variables que evalúan estilos de interacción familiar (autoritario, equilibrado y permisivo) los puntajes obtenidos son mayores y cercanos al centil 50, los cuales no fueron considerados como problemáticos si se tiene en cuenta la medida de dispersión de cada una de estas variables. 
Con relación a lo anterior, se considera que las familias en los tres niveles socioeconómicos se encuentran en términos de estilos autoritario, equilibrado y permisivo en un nivel promedio, respecto a la presencia y uso de cada uno en las diferentes situaciones presentadas en la prueba (ver Tabla 2).

En este grupo de padres, el estilo que más predomina es el equilibrado, seguido el autoritario y por ultimo se haya el estilo permisivo. Quienes presentan mayor nivel de estilo autoritario son los padres de nivel socio económico medio $(34,10)$ y bajo $(31,74)$, para el caso del estilo de interacción equilibrado, son las familias de nivel socio económico bajo $(52,46)$ y medio $(47,81)$ quienes presentan niveles más altos. El estilo permisivo, es el estilo más presente en las familias de nivel medio, siendo baja la presencia en las familias de nivel socioeconómico bajo $(11,88)$ y alto $(11,94)$

Tabla 2. Descripción de los estilos de interacción parental y nivel socioeconómico de las familias participantes.

\begin{tabular}{|c|c|c|c|c|}
\hline & & $\begin{array}{c}\text { Estilo } \\
\text { Autoritario }\end{array}$ & $\begin{array}{c}\text { Estilo } \\
\text { Equilibrado }\end{array}$ & $\begin{array}{c}\text { Estilo } \\
\text { Permisivo }\end{array}$ \\
\hline \multirow{5}{*}{ 至 } & P 25 & 18,00 & 44,00 & 32,00 \\
\hline & P 50 & 34,00 & 51,00 & 39,00 \\
\hline & P 75 & 46,00 & 62,00 & 44,00 \\
\hline & Media & 31,74 & 52,46 & 11,88 \\
\hline & DT & 14,14 & 11,72 & 1,37 \\
\hline \multirow{5}{*}{ 步 } & P 25 & 21,00 & 43,00 & 33,50 \\
\hline & P 50 & 39,00 & 47,00 & 42,00 \\
\hline & P 75 & 43,50 & 52,00 & 45,00 \\
\hline & Media & 34,10 & 47,81 & 50,00 \\
\hline & DT & 13,26 & 7,65 & 7,59 \\
\hline \multirow{5}{*}{ 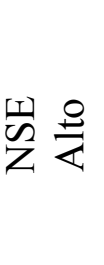 } & P 25 & 15,75 & 43,25 & 20,75 \\
\hline & P 50 & 30,50 & 49,00 & 31,00 \\
\hline & P 75 & 37,00 & 54,75 & 42,99 \\
\hline & Media & 27,69 & 11,92 & 11,94 \\
\hline & DT & 12,57 & 0,87 & 1,26 \\
\hline
\end{tabular}

DT Desviación típica P centil NSE Nivel socio económico

Tras realizar la prueba paramétrica ANOVA, se encuentran diferencias significativas entre las familias de nivel bajo y alto respeto al estilo de interacción permisivo $(p<.001)$ al igual que en las familias de nivel medio y alto $(\mathrm{p}<.05)$. No se evidencia diferencias significativas entre las familias que se caracterizarton por tener un estilo familiar autoritario y permisivo según el nivel socioeconómico. (Ver Tabla 3). 
Tabla 3. Comparación de los estilos de interacción familiar y nivel socioeconómico.

\begin{tabular}{|c|c|c|c|c|c|c|}
\hline & \multirow[t]{2}{*}{ Variable } & \multicolumn{2}{|c|}{ Anova } & \multirow{2}{*}{$\begin{array}{c}\text { Turkey } \\
\text { bajo-medio } \\
p \\
\end{array}$} & \multirow{2}{*}{$\begin{array}{c}\text { Turkey } \\
\text { bajo-alto } \\
p \\
\end{array}$} & \multirow{2}{*}{$\begin{array}{c}\text { Turkey } \\
\text { medio-alto } \\
p\end{array}$} \\
\hline & & gl & $p$ & & & \\
\hline \multirow{3}{*}{ 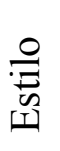 } & Autoritario & 2 & .121 & .733 & .410 & .104 \\
\hline & Equilibrado & 2 & .084 & .497 & .869 & .565 \\
\hline & Permisivo & 2 & $.001 * *$ & .945 & $.002 * *$ & $.004^{*}$ \\
\hline
\end{tabular}

\section{Habilidades sociales}

Se observa que en los componentes que evalúan las seis habilidades sociales (básicas de interacción social, para hacer amigos y amigas, conversacionales, relacionadas con los sentimientos, emociones y opiniones, de resolución de problemas interpersonales y para relacionarse con los adultos), los puntajes obtenidos teniendo en cuenta el género y la edad respecto al centil 50 no fueron considerados como problemáticos si se tiene en cuenta la medida de dispersión de cada una de estas variables.

Con relación a lo anterior podemos considerar que los niños y las niñas de esta investigación, en términos de desempeño en habilidades sociales, se encuentran en un nivel promedio, requiriendo desarrollar destrezas sociales que serán alcanzadas mediante las experiencias que los padres propicien en el contexto familiar (ver Tabla 4). Las habilidades que presentan un nivel de mayor desempeño en los niños y las niñas son las habilidades básicas de interacción social, seguida de las habilidades para relacionarse con los adultos.

$\mathrm{Al}$ comparar las seis habilidades sociales de los niños y las niñas respecto al género no se observan diferencias significativas $\left({ }^{* *} \mathrm{p}<.001,{ }^{*} \mathrm{p}<.05\right)$, indicando que el desempeño en las habilidades: de interacción, las conversacionales, para hacer amigos, para solucionar problemas interpersonales, relacionadas con los sentimientos y para interactuar con los adultos, no se explica por ser niño o niña (Tabla 5). 
Tabla 4. Descripción de habilidades sociales de los niños y niñas participantes según edad y género.

Habilidades Sociales

\begin{tabular}{|c|c|c|c|c|c|c|c|}
\hline & & $\begin{array}{l}\text { Básicas de } \\
\text { interacción } \\
\text { social }\end{array}$ & $\begin{array}{c}\text { Para } \\
\text { hacer } \\
\text { amistades }\end{array}$ & $\begin{array}{l}\text { De Conver- } \\
\text { sación }\end{array}$ & $\begin{array}{l}\text { Relacionada } \\
\text { con los sen- } \\
\text { timientos }\end{array}$ & $\begin{array}{l}\text { De resolución } \\
\text { de problemas } \\
\text { interpersonales }\end{array}$ & $\begin{array}{l}\text { Para rela- } \\
\text { cionarse } \\
\text { con adultos }\end{array}$ \\
\hline \multirow{5}{*}{ 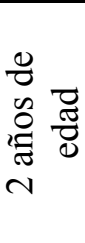 } & P 25 & 25,00 & 22,00 & 19,00 & 21,00 & 13,00 & 23,00 \\
\hline & P 50 & 31,00 & 28,00 & 24,00 & 26,00 & 19,00 & 27,00 \\
\hline & P 75 & 39,00 & 33,00 & 30,00 & 32,00 & 25,00 & 35,00 \\
\hline & Media & 31,22 & 21,69 & 24,79 & 25,64 & 19,40 & 28,22 \\
\hline & DT & 9,23 & 7,36 & 8,29 & 7,48 & 7,99 & 9,56 \\
\hline \multirow{5}{*}{ 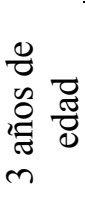 } & P 25 & 23,50 & 22,00 & 18,50 & 17,50 & 16,00 & 22,50 \\
\hline & P 50 & 33,00 & 29,00 & 25,00 & 25,00 & 20,00 & 28,00 \\
\hline & Р 75 & 38,00 & 34,00 & 34,00 & 34,00 & 26,50 & 36,00 \\
\hline & Media & 31,34 & 27,63 & 25,68 & 25,51 & 21,22 & 29,12 \\
\hline & DT & 9,55 & 7,90 & 9,63 & 8,63 & 9,38 & 1,73 \\
\hline \multirow{5}{*}{ 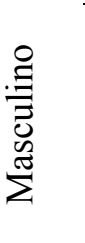 } & P 25 & 22,75 & 21,00 & 16,75 & 18,75 & 13,00 & 22,25 \\
\hline & P 50 & 30,50 & 26,50 & 23,00 & 24,00 & 19,00 & 26,00 \\
\hline & P 75 & 38,25 & 31,00 & 30,50 & 31,00 & 25,00 & 35,25 \\
\hline & Media & 30,09 & 26,00 & 23,89 & 24,44 & 19,50 & 27,31 \\
\hline & DT & 9,29 & 7,69 & 9,03 & 8,03 & 8,07 & 9,57 \\
\hline \multirow{5}{*}{ 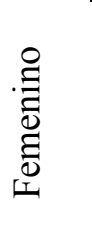 } & P 25 & 25,75 & 23,75 & 19,00 & 22,00 & 15,75 & 24,00 \\
\hline & P 50 & 31,50 & 29,00 & 25,00 & 26,50 & 19,50 & 28,00 \\
\hline & P 75 & 39,00 & 35,25 & 33,00 & 32,00 & 25,00 & 35,25 \\
\hline & Media & 32,44 & 29,33 & 26,37 & 26,74 & 20,68 & 29,81 \\
\hline & DT & 9,27 & 7,06 & 8,43 & 7,66 & 9,04 & 9,06 \\
\hline
\end{tabular}

DT Desviación Típica P centil

Tabla 5. Diferencias en habilidades sociales entre niños y niñas participantes según el género

\begin{tabular}{|c|c|c|c|c|c|}
\hline & \multirow[b]{2}{*}{ Variable } & \multirow{2}{*}{$\begin{array}{l}\text { Media } \\
\text { Niños }\end{array}$} & \multirow{2}{*}{$\begin{array}{l}\text { Media } \\
\text { Niñas }\end{array}$} & \multicolumn{2}{|c|}{ Género } \\
\hline & & & & $\mathrm{t}$ & $p$ \\
\hline \multirow{6}{*}{ 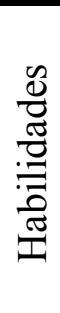 } & Básicas de interacción social & 30,09 & 32,44 & $-1,317$ & .725 \\
\hline & Para hacer amigos y amigas & 26,00 & 29,33 & $-2,347$ & .482 \\
\hline & Conversacionales & 23,89 & 26,37 & $-1,475$ & .701 \\
\hline & Relacionada con los sentimientos & 24,44 & 26,74 & $-1,520$ & .599 \\
\hline & De resolución de problemas interpersonales & 19,50 & 20,68 & $-0,719$ & .965 \\
\hline & Para relacionarse con los adultos & 27,31 & 29,81 & $-1,394$ & .786 \\
\hline
\end{tabular}

$* * \mathrm{p}<.001 * \mathrm{p}<.05$ 
Relaciones existentes entre los estilos de interacción parental y las habilidades sociales

La Tabla 6 muestra los resultados del análisis de correlación entre las variables de estudio. Se observa que las correlaciones obtenidas entre los estilos de interacción parental y las habilidades sociales fueron las siguientes:

El estilo autoritario se correlaciona negativamente con las habilidades básicas de interacción; lo cual dio una relación inversa, ya que entre más estrategias de contención autoritarias estén presentes en los padres, menos desarrollo de habilidades para relacionarse con los demás se harán presentes en los niños y las niñas. En esta misma línea investigativa, se relaciona este estilo con las habilidades para hacer amigos y amigas, habilidades conversacionales, habilidades relacionadas con los sentimientos, emociones y opiniones y habilidades para relacionarse con los adultos.

Tabla 6. Correlación entre los estilos de interacción parental y las habilidades sociales de los niños y niñas.

\begin{tabular}{|c|c|c|c|c|c|c|}
\hline \multicolumn{7}{|c|}{ Habilidades Sociales } \\
\hline & $\begin{array}{l}\text { Básicas de } \\
\text { Interacción }\end{array}$ & $\begin{array}{c}\text { Para } \\
\text { hacer } \\
\text { amigos } \\
\text { y ami- } \\
\text { gas }\end{array}$ & Conversacionales & $\begin{array}{l}\text { Relacionadas } \\
\text { con los } \\
\text { sentimientos, } \\
\text { emociones y } \\
\text { opiniones }\end{array}$ & $\begin{array}{l}\text { De solución de } \\
\text { problemas } \\
\text { interpersonales }\end{array}$ & $\begin{array}{l}\text { Para relacio- } \\
\text { narse con los } \\
\text { adultos }\end{array}$ \\
\hline Autoritario & $-.266 * *$ & $-.266^{* *}$ & $-.218^{*}$ & $-.362 * *$ & -.160 & $-.216^{*}$ \\
\hline Externo & $-.306^{* *}$ & $-.264 * *$ & $-.239 *^{*}$ & $-.402 * *$ & $-.196 * *$ & $-.275^{* *}$ \\
\hline Interno & $-.138^{*}$ & $-.126^{*}$ & $-.210 *$ & $-.239 *$ & $-.093^{*}$ & -.115 \\
\hline $\begin{array}{l}\text { Trasgresión } \\
\text { de la norma }\end{array}$ & $-.285^{* *}$ & $-.274 * *$ & $-.221 * *$ & $-.274 * *$ & $-.180^{* *}$ & $-.252 * *$ \\
\hline Equilibrado & .220 & $.334 * *$ & $.329 * *$ & $.299 * *$ & 0,056 & $.196^{*}$ \\
\hline Externo & $.098^{* *}$ & $.209^{*}$ & $-.226^{*}$ & .169 & $.011^{* *}$ & $.073^{*}$ \\
\hline Interno & .183 & $.339 * *$ & $.325 * *$ & $.320 * *$ & .149 & $.252 * *$ \\
\hline $\begin{array}{l}\text { Trasgresión } \\
\text { de la norma }\end{array}$ & $.199 * *$ & $.291 * *$ & $.207^{*}$ & $.223 * *$ & -.031 & $.197 *$ \\
\hline Permisivo & -.131 & -.145 & $-.194 *$ & -.111 & -.061 & -.083 \\
\hline Externo & -.053 & -.035 & -.145 & -.124 & -.063 & -.059 \\
\hline Interno & $-.238^{*}$ & $-.215^{*}$ & $-.195^{*}$ & -.089 & -.048 & -.115 \\
\hline $\begin{array}{l}\text { Trasgresión } \\
\text { de la norma }\end{array}$ & -.074 & -.090 & -.145 & -.048 & -.042 & -.072 \\
\hline
\end{tabular}

Respecto al estilo equilibrado se observa cuatro relaciones directas con las habilidades para hacer amigos y amigas, habilidades conversacionales, habilidades relacionadas con los 
sentimientos emociones y opiniones y habilidades para relacionarse con los adultos. Esto indica que a mayor presencia de estrategias y estilos de interacción equilibrado en los padres, mayores niveles de desarrollo de cada una de estas habilidades tienen los niños y las niñas.

Finalmente, el estilo permisivo se correlaciona negativamente con las habilidades conversacionales, es decir, que entre más permisividad por parte de los padres menor es la habilidad y repertorio conversacional de los niños y las niñas.

\section{Discusión y Conclusiones}

Desde una mirada multidimensional del desarrollo se reconoce y se da importancia a que el niño y la niña se encuentran perneados no sólo por componentes genético-hereditarios, sino, a su vez, por componentes socioculturales, donde se le da un papel primordial a los padres; y más específicamente, a como estos se vinculan con sus hijos e hijas. Del tipo relacional que se establece hijas se desprende y genera aprendizajes y avances en el desarrollo de cada una de las dimensiones humanas (social, cognitiva, comunicativa, afectivo-emocional, estética, ética-espiritual y corporal). Para Vasco y Henao (2008) lo genético no precisamente determina el desarrollo humano, es con lo social y la presencia del lenguaje que se despliega este; en otras palabras, mediante las experiencias sociales y culturales, el componente genético entra a formar parte de su constitución como ser humano. Son varios los aspectos que involucran a la familia como el principal agente contextual del desarrollo humano; los estilos de interacción que los padres y los adultos cercanos a los niños ponen de manifiesto en el contexto familiar repercuten en el desarrollo de estos.

Las familias de este estudio, fueron consideradas como el primordial agente contextual del desarrollo social; están presentan características propias de los tres estilos de interacción parental: autoritario, equilibrado y permisivo; se hacen evidentes a través de acciones y verbalizaciones que surgen como estrategias para encausar los comportamientos de los hijos. Estos estilos no están presentes de manera pura, sino que emergen de acuerdo a los aprendizajes y bagajes socioculturales en que han estado inmersas las familias, y sobretodo de las situaciones particulares que experimentan en el contacto con los infantes; es así, como un padre puede presentar acciones orientadas a una estructura autoritaria cuando su hijo comete una falta contra los valores familiares; pero puede interactuar con él de manera más comunicativa y empá- 
tica cuando este presentan una dificultad de desempeño emocional. Aunque varíen en intensidad o frecuencia, los estilos presentan un impacto, el cual actúa independiente de las características psicológicas que tengan los niños (Darling y Steinberg, 1993; Maccoby y Martin, 1983).

Respecto a las habilidades sociales o conductas necesarias para interactuar y relacionarse con los iguales y con los adultos de forma efectiva y mutuamente satisfactoria presentes en los niños y las niñas evaluados, se encuentra que los seis grupos de habilidades están presentes en todos los niños y las niñas, existiendo diferencias entre los niños y las niñas pertenecientes a un grupo familiar con unos estilos de interacción determinados. Se resalta que los padres con estrategias más equilibradas permiten mayor desarrollo de estas habilidades que padres con estructuras permisivas y autoritarias (Monjas, 2000; Zabala, Valadez, y Vargas, 2008).

$\mathrm{Al}$ analizar las asociaciones existentes entre el desarrollo de habilidades sociales de los niños y las niñas con relación al estilo de interacción parental de los progenitores, se logran hallazgos que van en concordancia con los resultados presentes en diversos estudios que han investigado las acciones o estilos de interacción de los padres y la manera como estos se relacionan con el desarrollo emocional y social. Padres y madres que crean y hacen uso de estrategias equilibradas mediadas por el dialogo, manifestaciones de afecto y normas establecidas propician en sus hijos e hijas aprendizajes de repertorios sociales más amplios, por el contrario aquellos padres y madres que se relacionan con sus hijos e hijas desde acciones más punitivas, rígidas y autoritarias, o a la inversa desde estrategias pobres en control y bastas en afecto y permisividad, generan en sus hijos e hijas comportamientos disfuncionales y pobres en aprendizajes adecuados para su edad y contexto (Ballesteros, 1995; Chang y Dodge, 2003; Cowan et al., 1996; Eisenberg et al., 2001; Florsheim et al., 1996; Patterson et al., 1992; Rogers y Holmberck, 1997; Shaw et al., 1994; Stoolmiller, 2001).

Quizá las investigaciones más conocidas sobre los estilos de interacción familiar son los estudios de Baumrind (1967, 1971), aunque ella los denomina estilos parentales. Las hipótesis plateadas por Baumrind $(1971,1973)$, acerca de la asociación existente entre la relación parental y algunas características sociales y emocionales de los niños y las niñas, son un punto de análisis y comparación de esta investigación, al igual que el estudio de García y Román (2003) y Henao y García (2009). Estos estudios se desarrollaron en relación a las acciones de 
los padres, centrándose en los estilos parentales, en la forma en que los padres despliegan los procesos relacionados con la norma y el manejo de autoridad en sus hijos e hijas. En estos se consideran que los padres autoritarios son aquellos que presentan un patrón muy prohibitivo de crianza, caracterizado por la presencia de muchas reglas y uso de tácticas disciplinarias fuertes. Los padres equilibrados son controladores pero flexibles, son sensibles hacia las necesidades y particularidades de los niños y las niñas, pero a la vez proporcionar parámetros para obedecer los límites que establecen. Los padres permisivos sobresalen por su accionar aceptador y laxo, por una conducción vaga de la norma y una manifestación alta de afecto y protección. En el desarrollo de esta investigación, se retoman las hipótesis plateadas por los autores mencionados, quienes establecieron vínculos entre la influencia de la relación parental y algunas características sociales y emocionales de los niños y las niñas, que se instituyen a partir del tipo de comunicación y normatización utilizado dentro del contexto familiar.

Las prácticas y hábitos de crianza de los padres, la respuesta que ofrecen hacia las demandas y necesidades de sus hijos e hijas, el afecto que les brindan, las formas de control y el tipo de disciplina que manejan, son estrategias de socialización que usan los padres para regular el comportamiento y potenciar el desarrollo de sus hijos e hijas. Dependiendo de las acciones y estrategias familiares creadas por los padres y madres se generan ambientes estimulantes del desarrollo (Martinez, Inglés, Piqueras, y Ramos, 2010).

\section{Habilidades sociales y padres con estilo de interacción autoritario}

Los hallazgos de este estudio coinciden con los resultados propuestos por Fernández (2003) y Garcia, Ramirez y Lima (1998). El primero plantea que los padres autoritarios limitan el desarrollo cognoscitivo y socio-emocional de los hijos y las hijas; los padres coartan la capacidad de creatividad, convirtiéndolos en reprimidos, callados e inseguros. Los segundos, encontraron en su estudio que los padres al transmitir de manera impositiva y arbitraria los mensajes, utilizando la intimidación verbal, física y de privaciones, consiguen la aceptación de lo que se dice por parte de los niños y las niñas, pero de una manera sumisa, alterando el desarrollo social. Los padres y madres de nuestro estudio, por buscar una inmediatez en las respuestas de sus hijos e hijas, sin considerar sus necesidades, y emitir ante cualquier falla una evaluación y acción de sanción, generan límites para el encuentro favorable con los amigos, amigas y adultos. Sus hijos se caracterizan por pobres repertorios de interacción, lo que dificulta el inicio y mantenimiento de conversaciones, actividades escolares, grupales y de juego; 
presentan niveles de ansiedad en situaciones sociales desconocidas, asumen posturas sumisas ante cualquier figura de autoridad, esto se relaciona con la presencia de pocas estregarías de afrontamiento y solución de problemas interpersonales. Las demandas de las figuras paternas son producidas de forma instantánea sin tener en cuenta las necesidades del niño; lo cual se relaciona con los pocos repertorios identificados en él para tomar hábitos y rutinas dentro del contexto familiar (Baumrind, 1972).

Estos padres resultan ser prohibitivos, se caracterizan por la imposición de muchas reglas, donde sus expectativas se centran en recibir una obediencia estricta, sin necesidad de una explicación previa o posterior a los actos. Esta estructura se convierte en un bloqueador o limitante del desarrollo de habilidades sociales conversacionales y repertorios esenciales de interacción, ya que no hay una exigencia o estimulación de expresión y dialogo con el niño y la niña. Estos niños se muestran en otros espacios sociales callados, temerosos y desconfiados de sus propias capacidades, son vigilantes de las normas, de su acatamiento y posibles sanciones; pero la lectura de estas normas se hace para una evitación del castigo y no desde el entendimiento de la importancia de estas para su desarrollo. En algunos casos, estos niños y niñas se muestran esquivos a las normas y rebeldes, buscando en otros espacios romper con las acciones punitivas de los padres. Los padres autoritarios no admiten niveles sensibles respecto a los puntos de vista en conflicto del niño o la niña, sólo esperan que este o esta acepten todo como ley; esto podría ser la génesis de posteriores dificultades en el desarrollo social de los niñas y las niñas, propiamente en las habilidades de auto-expresión, autoafirmación o asertividad, ya que a sus hijos e hijas no se les proporcionaría espacios de debate, argumentación o defensa, sólo y una afirmación constante del otro referente de autoridad. Así mismo, no se estimulan las habilidades cognitivo- sociales necesarias para la resolución de conflictos relacionados con problemas interpersonales (Monjas, 2000).

\section{Habilidades sociales y padres con estilo de interacción permisivo}

Los estilos de crianza de sobreprotección y favoritismo se relacionan con baja amabilidad y baja apertura (Aluja, Del Barrio y García, 2007). Para Fernández (2003) los padres permisivos restringen el desarrollo cognoscitivo y socio-emocional de los hijos y las hijas; propician el desarrollo de niños y niñas agresivos, con bajos logros escolares y dependientes. Conclusiones que van en la misma dirección de los resultados de nuestro estudio. El desempeño social presente en los niños y niñas de padres y madres que se relacionan desde pautas 
flexibles y permisivas, presenta pocos repertorios a nivel de conversacional, ya que la inmediatez y bajos niveles de tolerancia los llevan a no respetar la palabra del otro, a no defender sus puntos de vistas desde argumentaciones y diálogos amables y a no hacer lectura de las emociones e interés del otro.

Un grupo de padres y madres de este grupo se caracterizan por un predominio de laxitud, donde las exigencias se desplazan al otro punto, que quien exige y demanda son los hijos. Estos padres presentan un control parental escaso y flexible, y en ocasiones ausente; además, poseen una comunicación unidireccional y poco efectiva (Garcia, Ramirez y Lima, 1998). Los padres consienten que sus hijos expresen con libertad sus sentimientos e impulsos, quienes no desarrollan bases para estructurar habilidades conversacionales (iniciar conversación, mantener conversaciones, terminar conversaciones, unirse a la conversación de otros, y conversaciones de grupo) acordes a su edad y a las convenciones contextuales. Los padres no supervisan las actividades y rara vez ejercen un control firme sobre su comportamiento, presentan una imposibilidad de manejar e imponer la norma al superponer los deseos del niño, de ahí que las habilidades básicas de interacción como sonreír y reír, saludar, presentarse, pedir favores, y ser cortes y amable, no sean altamente estimuladas.

Estos resultados coinciden con Baumrind (1967, 1968 y 1970), en sus estudios, ella concluye que los niños ansiosos, inquietos y deprimidos se asocian con padres que ejercían menos control y exigencia de madurez, se comunicaban menos y mostraban menor apoyo.

\section{Habilidades sociales y padres con estilo de interacción equilibrado}

Los resultados de esta investigación, coinciden con los hallazgos de diversos autores que han estudiado las prácticas y estilos de interacción parental de los padres en asociación con el desarrollo emocional y social y de los hijos e hijas (Asili y Prats, 2002; Boyes y Allen, 1993; Cakir y Aydin, 2005; Garcia y Román, 2003; Marks y Mclanahan, 1993; Scoutt y Scoutt, 1991). Al respecto, los estudios clásicos de Baumrind; establecen la relación entre el accionar de los padres y los patrones de personalidad de sus hijos, ella concluye que los niños y las niñas que manifiestan niveles altos de confianza en sí mismos son hijos e hijas de padres que revelan alto grado de presencia en el hogar, de comunicación y de apoyo, con presencia de una normativa clara. Este tipo de padres se catalogan como equilibrados. Al igual que los hallazgos obtenidos por Baumrind $(1971,1972)$ y García y Román (2003) resal- 
tan el estilo equilibrado como el generador de mejores niveles en el desempeño infantil a nivel socioemocional.

Los padres de nuestro estudio que manifestaron la presencia de estrategias equilibradas en diversas situaciones, propician en sus hijos e hijas una presencia mayor de habilidades sociales en comparación con los padres que presentan acciones autoritarias y permisivas.

Los padres que se enmarcan en el estilo de interacción equilibrado, se caracterizan por un estilo examinador pero a la vez flexible, sus exigencias están mediadas por un reconocimiento y lectura de las demandas y necesidades de sus hijos e hijas. Los niños y las niñas con padres equilibrados de nuestro estudio, manifiestan repertorios básicos para relacionarse con las otras personas, conductas para entablar un contacto adaptativo con los pares y los adultos, y destrezas acordes a su edad que les permiten iniciar, mantener y finalizar conversaciones con otras personas, estos resultados coinciden con los hallazgos de Mestre, Tur, Samper, Nácher y Cortés (2007). Estos niños y niñas ante problemas de índole interpersonal (peleas, rechazo social, mal entendidos, discrepancias, etc) acuden a estrategias de solución mediadas por el dialogo y la conciliación, donde sus intereses se median con los de los demás. Hacen lectura de problemas sociales, identificando posibles causas y construyendo estrategias de solución acurdes a su edad y posibilidades.

Estos padres crean cimientos para hacer respetar los límites de una manera estable, manifiestan una apertura y sensibilidad hacia la participación activa de sus hijos e hijas y buscan su colaboración en la toma de decisiones familiares, lo que posibilita que sus hijos e hijas presenten conductas sociales que favorezcan el desarrollo posterior de la habilidades relacionadas con los sentimientos, las emociones y las opiniones, también llamadas auto-expresión, autoafirmación o asertividad (Baumrind, 1967). En este sentido padres posibilitadores de verbalizaciones emocionales positivas estimulan en los niños y las niñas la presencia en años posteriores de una asertividad que conlleve la expresión y defensa de los propios sentimientos y derechos, considerando y respetando los de los demás (Monjas, 2000), a la vez que logran apropiarse de las normas establecidas en los distintos espacios sociales de en los que participan.

En este tipo de estilo relacional prima la expresión de afectos, a la vez que se establecen normas y límites. Se generan puentes de comunicación, de afecto y de limites que estimu- 
lan el desarrollo de habilidades básicas de interacción, estas se hacen evidentes en el desempeño que presentan los niños y niñas en el contexto familia, escolar y social, ellos y ellas acuden a gestos, verbalizaciones y acciones de cortesía manifiestas en el momento de saludar y despedirse, y cuando necesitan solicitar una ayuda o brindar atención a otros. Un ambiente familiar equilibrado permite que los niños y niñas se integren con mayor facilidad a los grupos de pares, resaltando estos como lideres al ser quienes toman iniciativa en las interacciones y juegos, a la vez que refuerzan las conductas de los compañeros y apoyan desde acciones cooperativas y colaborativas fines comunes. Estos niños y niñas se distinguen por relacionarse de manera eficaz con los adultos (docentes, familiares, vecinos, etc), respetándolos como autoridad pero estableciendo diálogos tranquilos y directos; a su vez, estos niños y niñas hacen lecturas de índole social y emocional; identifican situaciones positivas y negativas presentes en lugares y personas y desde allí dan respuesta y actúan. En síntesis, los padres equilibrados o democráticos según Fernández (2003), forman niños y niñas seguros, independientes, adaptados socialmente y exitosos.

Nuestra investigación permitió considerar que aquellos padres y madres que presentan un control que es mediado por muestras de afecto y comprensión hacia sus hijos e hijas, logran el desarrollo de niños y niñas con mayores habilidades sociales que les permiten participar con mayor facilidad de encuentros de interacción con diversas personas y en distintas situaciones, y actuar acorde a cada una de los eventos generados. Esto va de acuerdo con lo que Garcia, Ramirez y Lima (1998). Plantearon, ya que para ellos los estilos educativos parentales inciden positiva o negativamente en la adquisición de valores prosociales, de auto dirección, y en el logro de aprendizajes significativos en los niños y las niñas.

En resumen, el presente estudio muestra relaciones entre algunas características de los estilos de interacción parental y el desarrollo de ciertas habilidades sociales. Estas relaciones sugieren cómo la presencia de un estilo equilibrado interviene y favorece el desarrollo de habilidades sociales, y cómo los estilos autoritario y permisivo, disminuyen el desarrollo de estas habilidades en los niños y las niñas. Padres democráticos, que logran leer y comprender las características de sus hijos e hijas, que se relacionan desde vínculos afectivos, donde los derechos y deberes prevalecen, y que crean espacios enriquecidos de expresión, toma de decisión e independencia, permiten a los niños y niñas lograr aprender repertorios amplios de socialización, y con ella una competencia y desempeño social. Por el contrario, padres rígidos, estrictos y ajenos a las necesidades de los niños y las niñas, para quienes prima el logro y 
cumplimiento de reglas, sin tener de por medio la comunicación y el dialogo; coartan y limitan ambientes que estimulen la generación de conductas de interacción y desenvolvimiento en situaciones sociales. Así mismo, padres laxos, que privilegian en los niños y las niñas el deseo sobre la responsabilidad, y que se vinculan sin límites claros; impiden actuaciones en los niños y las niñas propicias para interactuar con los otros y desempeñarse en ámbitos sociales.

Los resultados de este estudio aportan información relevante y pertinente sobre los factores asociados al comportamiento de niños y niñas desde la dimensión social, lo cual es punto de partida para la creación y aplicación de programas de formación para los padres.

El constructo estilo de interacción parental conlleva un amplio e importante análisis por la variedad de elementos que involucra y su relación en el desarrollo de los niños y las niñas. Aunque desde este estudio se destaquen las acciones de los padres como predictores fuertes de las disposiciones conductuales de los hijos e hijas, es necesario seguir profundizando en esta dimensión para poder obtener implicaciones directas e indirectas que permitan el diseño, evaluación y ejecución de programas de promoción, prevención e intervención con los padres.

Los estilos de interacción parental se definen como aquellas pautas o costumbres que se transfieren de generación en generación en una cultura, refiriéndose a la forma como los padres y adultos cuidan y educan a sus hijos e hijas. Es así, como los estilos de interacción dependen de los aprendizajes obtenidos en la propia crianza de los padres y de la influencia cultural.

Los niños y las niñas con padres que presentan un estilo de interacción equilibrado muestran un mejor desempeño social, mientras que los hijos e hijas con padres permisivos y autoritarios generan menor desarrollo de cada una de las habilidades sociales requeridas para generar una competencia social. Los hallazgos obtenidos en esta investigación afirman lo encontrado por Garcia y Román (2005), quienes en su investigación evaluaron las prácticas educativas familiares, obteniendo como resultado que los padres equilibrados que hacían una lectura de las necesidades de sus hijos e hijas desde la presencia de exigencias y responsabilidades fundaban en sus hijos e hijas altos niveles de autoestima. 
Este estudio resalta la importancia de la acción de los padres, madres y cuidadores en el desarrollo social de los niños y niñas en los diferentes niveles socio-económicos, resulta fundamental identificar elementos diferenciadores de los estilos de interacción y desempñeo social según la estructura familiar, el nivel educativos de los cuidadores, la presencia de hermanos y la escolaridad de los niños y niñas, lo que permita comprender los factores familiares estimulantes del desarrollo social. Este estudio es una base para estudios posteriores que se realicen en el contexto familiar y el desarrollo en la primera infancia.

\section{Referencias}

Aluja, A., del Barrio, V., y García, L. F. (2007). Personalidad, valores sociales y satisfacción de pareja como factores predictores de los estilos de crianza parentales. International Journal of Clinical and Health Psychology, 7, 725-737.

Asili P. N. y Pinzón, B. K. (2003). Relación entre estilos parentales, estilos de apego y bienestar psicológico. Psicología y Salud, 13(2), 215-225.

Asili P., N., y Prats B., R.L. (2002). Percepción de estilos parentales y bienestar psicológico. Psicología y Salud, 12(2), 179-188

Ballesteros, B.P. (1995). El conflicto marital y su relación con problemas de ajuste con los niños. Bogotá: Fundación Universitaria Konrad Lorenz

Baumrind, D. (1967). Current Patterns of parental authority. Developmental Psychology, 28(30), 421-424.

Baumrind, D. (1968). Authoritarian vs. authoritative parental control. Adolescence, 31, 255271.

Baumrind, D. (1970). Socialization and instrumental competence in young children. Children, 26(2), 104-119.

Baumrind, D. (1971). Current patterns of parental authority. Developmental Psychology Monograph, 4, 1-103.

Baumrind, D. (1972). Manual f or the Primary School Behavior Q-sort. Institute of Human Development, University of California, Berkeley.

Baumrind, D. (1973). The development of instrumental competence trough Socialization. En A. D Pick (Ed), Minnesota Sympostum on child psychology (pp. 3-46). Minneapolis: University of Minnesota Press.

Boyes, M., y Allen, S. (1993). Patterns of parent-child interaction and socio-moral reasoning in adolescence and young adulthood. Merrill-Palmer Quarterly, 39(4), 551-570.

Bronfenbrenner, U. (1987). La ecología del desarrollo humano (1 ed.). Barcelona: Paidos

Caballo, V. E. (1993). Manual de evaluación y entrenamiento de las habilidades sociales. Madrid: Siglo Veintiuno. En M.I Monjas y B. González Moreno (1998). Las habilidades Sociales en el Curriculo. (pp. 146). España: Ministerio de Educación, Cultura y Deporte. Secretaría General de Educación y Formación Profesional. Centro de Investigación y Documentación Educativa. 
Cakir, S.G., y Aydin, G. (2005). Parental attitudes and Ego identity status in Turkish adolescents. Adolescence, 160, 848-859.

Ceballos, E. y Rodrigo, M. J. (1998). Las metas y estrategias de socialización entre padres e hijos. En M.J Rodrigo y J. Palacios (coords.) Familia y Desarrollo Humano (pp. 225244). Madrid: Síntesis

Chang, S. y Dodge, M. (2003). Harsh parenting in relation of child emotion regulation and aggression. Journal of family psychology. 17(4), 598-606.

Coll, C. (1988). Conocimiento psicológico y práctica educativa. Introducción a las relaciones entre psicología y educación. Barcelona: Barcanova.

Coll, C., Miras, M., Onrubia, J. y Solé, I. (1998). Psicología de la educación. Barcelona: Edhasa.

Cowan, P. A., Cohn, D. A., Cowan, C. P. y Pearson, J. L. (1996). Parents' attachment histories and childrens' externalizing and internalizing behaviors: exploring family systems models of linkage. Journal of Consulting and Clinical Psychology. 64(1), 53-63.

Darling, N. y Steinberg, L. (1993). Parenting style as context: An integrative model. Psychological Bulletin, 113, 487-496.

Eisenberg, N., Losoya, S., Fabes, R.A.,Guthrie, I.K., Reiser, M.,Murphy, B.,Shepard, S.A., Poulin, R. y Padgett, S.J. (2001). Parental socialization and childrens dysregulated expression of emotion and externalizing problems. Journal of family Psychology, 15(2), 183-205.

Eisenberg, N., Valiente, C., Morris, A. S., Fabes, R. A., Cumberland, A. y Reiser, M. (2003). Longitudinal relations among parental emotional expressivity, children's regulation, and quality of Socioemotional functioning. Developmental Psychology, 39(1), 3-19.

Elliot, S., y Gresham, F. (1991). Social skills intervention guide. Austin, TX: Pro-ed.

Fernández, I. (2003). Influencia de los estilos de paternidad en el Desarrollo cognoscitivo y socioemocional de los preescolares. Rey de Enseñanza e Investigación en Psicología, 6, 1-29.

Florsheim, P., Tolan, P. H. y Gorman-Smith, D. (1996). Family processes and risk for externalizing behavior problems among African American and Hispanic boys. Journal of Consulting and Clinical Psychology, 64(6), 1222-1230

Forgas, J. (2001). Feeling and thiking. The role of affect in social cognition. Cambridge University Paris: Press \&Edition de la Maison de sciences de I'Homme.

Garcia, M. D., Ramirez, G. y Lima, A. (1998). La construcción de valores en la familia. En M.J Rodrigo y Jesus Palacios (Coords). Familia y desarrollo humano (pp. 201-221). Madrid: Alianza Editorial.

García, J. y Román, J.M. (2003). Escala de Identificación de Prácticas Educativas Familiares. Madrid: CEPE, S.L

García, J. y Román, J.M. (2005). Practicas educativas familiares y autoestima. Psicothema, 17 (1), 76-82.

Henao, G.C y García, M.C. (2009). Interacción familiar y desarrollo emocional en niños y niñas. Revista Latinoamericana de Ciencias Sociales, Niñez y Juventud, 7(2), 785-802.

Hundert, J. (1995). Enhancing social competence in young students. Austin, TX: pro-ed. En M.I Monjas y B. González Moreno (1998). Las habilidades Sociales en el Curriculo. 
146. España: Ministerio de Educación, Cultura y Deporte. Secretaría General de Educación y Formación Profesional. Centro de Investigación y Documentación Educativa.

Maccoby, E. E. y Martin, J. A. (1983). Socialization in the context of the family: Parent-child interaction. Handbook of child psychology, 4, 1-101.

Marks, N. F., y Mclanahan, S. S. (1993). Gender, Family Structure, and Social Support among Parents Journal of Marriage and the Family, 55(2), 481-493.

Martinez, A.E, Inglés, C.J, Piqueras, J.A. y Ramos, VB. (2010). Importancia de los amigos y los padres en la salud y el rendimiento escolar. Electronic Journal of research in educational psychology, 20, 8(1), 111-138.

Mestre, M., Tur, A., Samper, P., Nácher, M. y Cortés, M. (2007). Estilos de crianza en la adolescencia y su relación con el comportamiento prosocial. Revista latinoamericana de psicología., 39(2), 211-225.

Monjas, M. I y González, B. (1998). Las habilidades Sociales en el Curriculo. En M.I Monjas y B. González Moreno (1998). Las habilidades Sociales en el Curriculo. Madrid: Ministerio de Educación, Cultura y Deporte.

Monjas, M. I. (2000) Programa de Enseñanza de Habilidades de Interacción Social (PEHIS) para niños y niñas en edad escolar Madrid: Ciencias de la Educación Preescolar y Especial CEPE

Patterson, G. R., Reid, J. B. y Dishion, T. J. (1992). Antisocial Boys. Eugene, OR: Castalia

Rogers, M. J. y Holmbeck, G. N. (1997). Effects of interparental aggression of childrens' adjustments the moderating role of cognitive appraisal and coping. Journal of family psychology March. 11(1), 125-130.

Rogoff, B. (1993). Aprendices del pensamiento. El desarrollo cognitivo en el contexto social. Barcelona: Paidos

Scoutt, W. A., y Scoutt, R. (1991). Family relationships and children's personality: A crosscultural, cross source comparison. British Journal of Social Psychology, 30, 1-20

Shaw, D. S., Keenan, S. y Vondra, J. I. (1994). Developmental Precursors of Externalizing behavior age 1 to 3. Developmental Psychology, 30(3), 355-364.

Solé, I. (1997). Les pràctiques educatives com a contextos de desenvolupament. En C. Coll, M. Miras, J. Onrubia, y I Solé (Coordina-dor). Psicologia de l'Educació (159-242). Barcelona: Ed. de la Universitat Oberta de Catalunya

Sorkhabi, N. (2005). Applicability of Baumrind's parent typology to collective cultures: Analysis of cultural explanations of parent socialization effects. International Journal of Behavioral Development, 29, 552-563.

Sroufe, A. (2000). Desarrollo emocional: la organización de la vida emocional en los primeros años. México: Oxford University Press

Stoolmiller, M. (2001). Synergistic interaction of child manageability problems and parentsdiscipline tactics in predicting future growth in externalizing behavior for boys. Developmental Psychology. 37(6), 814-825.

Vallejo, A., Aguilar, J. y Valencia, A. (2002). Estilos de paternidad en padres totonacas y promoción de autonomía psicológica hacia los hijos adolescentes. Psicología y Salud, 12(1), 101-108. 
Vasco, C. E. y Henao, G. C. (2008). Elementos y modelos del desarrollo: una revisión del concepto. En J. Larreamendi-Joerns, R. Puche-Navarro y A. Restrepo Ibiza (comps.), Claves para pensar el cambio: ensayos sobre psicología del desarrollo (pp. 1-28). Bogotá: Universidad de los Andes-Facultad de Ciencias Sociales-Ceso-Departamento de Psicología.

Vigotsky, L. (1979). El desarrollo de los procesos psicológicos superiores. Barcelona: Grijalbo

Vila, I. (1998). Familia, escuela y comunidad. Barcelona: Horsor

Zabala, M.A, Valadez, M.D y Vargas, M.C. (2008). Inteligencia emocional y habilidades sociales en adolesecntes con alta aceptación social. Electronic Journal of research in educational Psychology, 15, 6(2), 319-338. 